\title{
A new type fixed point theorem for a contraction on partially ordered generalized complete metric spaces with applications
}

\author{
Madjid Eshaghi Gordji ${ }^{1}$, Maryam Ramezani ${ }^{1}$, Farhad Sajadian' ${ }^{1}$, Yeol Je Cho ${ }^{2 *}$ and Choonkil Park ${ }^{3}$
}

${ }^{\text {*Correspondence: yjcho@gnu.ac.kr }}$
${ }^{2}$ Department of Mathematics
Education and the RINS,
Gyeongsang National University,
Chinju, 660-701, Korea
Full list of author information is
available at the end of the article

available at the end of the article

\begin{abstract}
In this paper, we prove a fixed point theorem for a contraction in generalized complete metric spaces endowed with partial order. As an application, we use the fixed point theorem to prove the Hyers-Ulam stability of the Cauchy functional equation in Banach spaces endowed with a partial order.

MSC: $54 \mathrm{H} 25 ; 47 \mathrm{H} 10 ; 39 \mathrm{~B} 52$

Keywords: fixed point; generalized complete metric space; partial order; Hyers-Ulam stability
\end{abstract}

\section{Introduction}

In 1940, Ulam gave a wide ranging talk in front of the mathematics club of University of Wisconsin in which he discussed a number of important unsolved problems (see [1]). One of the problems was the question concerning the stability of homomorphisms:

Let $G_{1}$ be a group and $G_{2}$ be a metric group with a metric $d(\cdot, \cdot)$. Given $\epsilon>0$, does there exist $\delta>0$ such that, if a mapping $h: G_{1} \rightarrow G_{2}$ satisfies the inequality $d(h(x y), h(x) h(y))<\delta$ for all $x, y \in G_{1}$, then there exists a homomorphism $H: G_{1} \rightarrow G_{2}$ with $d(h(x), H(x))<\epsilon$ for all $x \in G_{1}$ ?

In 1941, Hyers [2] affirmatively answered the question of Ulam for the case where $G_{1}$ and $G_{2}$ are Banach spaces. Taking this fact into account, the additive Cauchy functional equation $f(x+y)=f(x)+f(y)$ is said to satisfy the Hyers-Ulam stability.

On the other hand, Banach's contraction principle is one of the pivotal results of analysis. It is widely considered as the source of metric fixed point theory. Also, its significance lies in its vast applicability in a number of branches of mathematics. Many kinds of generalizations of the above principle have been a heavily investigated branch of research. In particular, Diaz and Margolis [3] presented the following definition and fixed point theorem in a 'generalized complete metric space'.

Definition 1.1 Let $X$ be an abstract (nonempty) set and assume that, in the Cartesian product $X \times X$, a distance function $d(x, y)(0 \leq d(x, y) \leq \infty$ for all $x, y \in X)$ is defined and satisfies the following conditions:

(D1) $d(x, y)=0$ if and only if $x=y$;

(D2) $d(x, y)=d(y, x)$ (symmetry);

(D3) $d(x, y) \leq d(x, z)+d(z, y)$ (triangle inequality);

@2014 Eshaghi Gordji et al.; licensee Springer. This is an Open Access article distributed under the terms of the Creative Commons Attribution License (http://creativecommons.org/licenses/by/2.0), which permits unrestricted use, distribution, and reproduction in any medium, provided the original work is properly cited. 
(D4) every $d$-Cauchy sequence in $X$ is $d$-convergent, i.e., $\lim _{m, n \rightarrow \infty} d\left(x_{n}, x_{m}\right)=0$ for a sequence $\left\{x_{n}\right\}$ in $X$ implies the existence of an element $x \in X$ with $\lim _{n \rightarrow \infty} d\left(x, x_{n}\right)=0$ (the point $x$ is unique by (D1) and (D3)).

Then we call $(X, d)$ a generalized complete metric space.

Theorem 1.2 Suppose that $(X, d)$ is a generalized complete metric space and the function $T: X \rightarrow X$ is a contraction, that is, $T$ satisfies the following condition:

(CI) There exists a constant $q$ with $0<q<1$ such that, whenever $d(x, y)<\infty$,

$$
d(T x, T y) \leq q d(x, y)
$$

Let $x_{0} \in X$ and consider a sequence $\left\{T^{l} x_{0}\right\}$ of successive approximations with initial element $x_{0}$. Then the following alternative holds: either

(A) for all $l \geq 0$, one has $d\left(T^{l}\left(x_{0}\right), T^{l+1}\left(x_{0}\right)\right)=\infty$

or

(B) the sequence $\left\{T^{l} x_{0}\right\}$ is d-convergent to a fixed point of $T$.

Recently, Nieto and Rodriguez-Lopez [4] proved a fixed point theorem in partially ordered sets as follows.

Theorem 1.3 Let $(X, \leq)$ be a partially ordered set. Suppose that there exists a metric $d$ in $X$ such that $(X, d)$ is a complete metric space. Let $f: X \longrightarrow X$ be a continuous and nondecreasing mapping such that there exists $k \in[0,1)$ with

$$
d(f(x), f(y)) \leq k d(x, y)
$$

for all $x, y \in X$. If there exists $x_{0} \in X$ with $x_{0} \leq f\left(x_{0}\right)$, then $f$ has a fixed point.

In 2003, Cădariu and Radu [5] applied the fixed point method to investigate the Jensen functional equation (see also [6-9]) and presented a short and simple proof (different from the direct method initiated by Hyers in 1941) for the Hyers-Ulam stability of the Jensen functional equation [5] for proving properties of generalized Hyers-Ulam stability for some functional equations in a single variable [7] for the stability of some nonlinear equations [6]. Recently, Brzdek [10], Brzdek and Cieplinski [11, 12] reported some interesting results in this direction (see also [13-16]).

In this paper, we prove a fixed point theorem for self-mappings on a partially ordered set $X$ which has a generalized metric $d$. Moreover, we give a generalization of the HyersUlam stability of the conditional Cauchy equation as an important result of our fixed point theorem.

\section{Main results}

We start our work by the following fixed point theorem in generalized complete metric spaces.

Theorem 2.1 Let $(X, d)$ be a generalized complete metric space and $\leq$ be a partial order on $X$. Let $f: X \rightarrow X$ be a continuous and nondecreasing mapping such that there exists 
$k \in[0,1)$ with

$$
d(f(x), f(y)) \leq k d(x, y)
$$

for all $x, y \in X$ with $x \geq y$. If there exists $x_{0} \leq f\left(x_{0}\right)$, then the following alternative holds: either

(A) for all $l \geq 0$, one has

$$
d\left(f^{l}\left(x_{0}\right), f^{l+1}\left(x_{0}\right)\right)=\infty
$$

or

(B) the sequence of $\left\{f^{n}\left(x_{0}\right)\right\}$ is d-convergent to a fixed point of $f$.

Proof Consider the sequence $\left\{d\left(f^{l}\left(x_{0}\right), f^{l+1}\left(x_{0}\right)\right)\right\}$ of real numbers. Then we consider two cases as follows:

(a) If, for all $l \geq 0, d\left(f^{l}\left(x_{0}\right), f^{l+1}\left(x_{0}\right)\right)=+\infty$, then (A) holds;

(b) If, for some integer $l, d\left(f^{l}\left(x_{0}\right), f^{l+1}\left(x_{0}\right)\right)<+\infty$, then $N=N\left(x_{0}\right)$ denotes the smallest nonnegative integer such that $d\left(f^{N}\left(x_{0}\right), f^{N+1}\left(x_{0}\right)\right)<+\infty$.

We see that $d\left(f^{N+1}\left(x_{0}\right), f^{N+2}\left(x_{0}\right)\right)<d\left(f^{N}\left(x_{0}\right), f^{N+1}\left(x_{0}\right)\right)<+\infty$ and, by induction, we have

$$
d\left(f^{N+l}\left(x_{0}\right), f^{N+l+1}\left(x_{0}\right)\right)<+\infty
$$

for all $l \geq 1$.

Note that $f$ is nondecreasing. Then we have

$$
x_{0} \leq f\left(x_{0}\right) \leq f^{2}\left(x_{0}\right) \leq \cdots \leq f^{n}\left(x_{0}\right) \leq \cdots
$$

and we can write $d\left(f^{n}\left(x_{0}\right), f^{n+1}\left(x_{0}\right)\right) \leq k d\left(f^{n}\left(x_{0}\right), f^{n-1}\left(x_{0}\right)\right)$ for all $n \geq 1$.

Now, by induction, we show that

$$
d\left(f^{n+1}\left(x_{0}\right), f^{N}\left(x_{0}\right)\right) \leq k^{n} d\left(f\left(x_{0}\right), x_{0}\right)
$$

for all $n>N\left(x_{0}\right)$. For $n=1$, since $x_{0} \leq f\left(x_{0}\right)$, we have

$$
d\left(f^{2}\left(x_{0}\right), f\left(x_{0}\right)\right) \leq k d\left(f\left(x_{0}\right), x_{0}\right) .
$$

Supposing that $(\Omega)$ holds for some $n$ and using that $f^{n}\left(x_{0}\right) \leq f^{n+1}\left(x_{0}\right)$, we obtain

$$
d\left(f^{n+2}\left(x_{0}\right), f^{n+1}\left(x_{0}\right)\right) \leq k d\left(f^{n+1}\left(x_{0}\right), f^{n}\left(x_{0}\right) \leq k k^{n} d\left(f\left(x_{0}\right), x_{0}\right)=k^{n+1} d\left(f\left(x_{0}\right), x_{0}\right) .\right.
$$

Thus it follows that $\left\{f^{n}\left(x_{0}\right)\right\}$ is a Cauchy sequence in $X$. Indeed, let $m>n>N\left(x_{0}\right)$. Then we have

$$
\begin{aligned}
d\left(f^{m}\left(x_{0}\right), f^{n}\left(x_{0}\right)\right) & \leq d\left(f^{m}\left(x_{0}\right), f^{m-1}\left(x_{0}\right)\right)+\cdots+d\left(f^{n+1}\left(x_{0}\right), f^{n}\left(x_{0}\right)\right) \\
& \leq\left(k^{m-1}+k^{m-2}+\cdots+k^{n}\right) d\left(f\left(x_{0}\right), x_{0}\right) \\
& =\frac{k^{n}-k^{m}}{1-k} d\left(f\left(x_{0}\right), x_{0}\right) .
\end{aligned}
$$


On the other hand, since $X$ is a complete generalized metric space, there exists $y \in X$ such that $\lim _{n \rightarrow \infty} f^{n}\left(x_{0}\right)=y$.

Finally, we prove that $y \in X$ is a fixed point of $f$, that is, $f(y)=y$. Let $\epsilon>0$ be a positive real number. Using the continuity of $f$ at $y$, for $\epsilon / 2$, there exists $\delta>0$ such that $d(z, y)<\delta$ implies that $d(f(z), f(y))<\epsilon / 2$. Now, by the convergence of $\left\{f^{n}\left(x_{0}\right)\right\}$ to $y$ and $\eta=\min \{\epsilon / 2, \delta\}>0$, there exists $n_{0} \in \mathbb{N}$ such that $n_{0}>N\left(x_{0}\right)$ and, for all $n \geq n_{0}, d\left(f^{n+1}\left(x_{0}\right), y\right)<\eta$. Therefore, for all $n>n_{0}$, we have

$$
d(f(y), y) \leq d\left(f(y), f\left(f^{n}\left(x_{0}\right)\right)\right)+d\left(f^{n+1}\left(x_{0}\right), y\right)<\epsilon / 2+\eta \leq \epsilon,
$$

and hence $f(y)=y$. This completes the proof.

Theorem 2.2 In Theorem 2.1, we can replace the following condition with the continuity off:

If $\left\{x_{n}\right\}$ is a nondecreasing sequence and $x_{n} \rightarrow x$ in $X$, then $x_{n} \leq x$ for all $n \in \mathbb{N}$.

Then $f$ has a fixed point.

Proof In Theorem 2.1, we just showed that $y$ is a fixed point of $f$. Let $\epsilon>0$ be given. Since $f^{n}\left(x_{0}\right) \rightarrow y$ and $\left\{f^{n}\left(x_{0}\right)\right\}$ is a nondecreasing sequence, we have $f^{n}\left(x_{0}\right) \leq y$. For any $\epsilon / 2>0$, there exists $n_{0} \in \mathbb{N}$ such that $n_{0} \geq l$ and, for all $n \geq n_{0}, d\left(f^{n_{0}}\left(x_{0}\right), y\right)<\epsilon / 2$. Therefore, we have

$$
\begin{aligned}
d(f(y), y) & \leq d\left(f(y), f^{n_{0}+1}\left(x_{0}\right)\right)+d\left(f^{n_{0}+1}\left(x_{0}\right), y\right) \\
& \leq k d\left(y, f^{n_{0}}\left(x_{0}\right)\right)+d\left(f^{n_{0}+1}\left(x_{0}\right), y\right) \\
& <k \epsilon / 2+\epsilon / 2 \\
& <\epsilon .
\end{aligned}
$$

This shows that $f(y)=y$. This completes the proof.

Remark 2.3 In Theorem 2.2, since $f^{n}\left(x_{0}\right) \rightarrow y$ and $\left\{f^{n}\left(x_{0}\right)\right\}$ is nondecreasing, we have $x_{0} \leq y$ and

$$
d\left(x_{0}, y\right) \leq d\left(x_{0}, f\left(x_{0}\right)\right)+d\left(f\left(x_{0}\right), f(y)\right) .
$$

Therefore, we have

$$
d\left(x_{0}, y\right) \leq d\left(x_{0}, f\left(x_{0}\right)\right)+k d\left(x_{0}, y\right) .
$$

Thus it follows that

$$
d\left(x_{0}, y\right) \leq \frac{1}{1-k} d\left(x_{0}, f\left(x_{0}\right)\right)
$$

Theorem 2.4 If, for all $x, y \in X$, there exists $z$ which is comparable to $x$ and $y$ and $d(z, x)<$ $\infty, d(z, y)<\infty$, then, in Theorems 2.1 and 2.2, the uniqueness of the fixed point off follows. 
Proof If $x \in X$ is another fixed point of $f$, then we prove that $d(x, y)=0$, where $y=$ $\lim _{n \rightarrow \infty} f^{n}\left(x_{0}\right)$. Since there exists $z \in X$ which is comparable to $x$ and $y, f^{n}(z)$ is comparable to $f^{n}(x)=x$ and $f^{n}(y)=y$ for all $n \in \mathbb{N} \cup\{0\}$ and

$$
d(x, y) \leq d\left(f^{n}(x), f^{n}(z)\right)+d\left(f^{n}(z)+f^{n}(y)\right) \leq k^{n} d(x, z)+k^{n} d(z, y) \rightarrow 0
$$

whenever $n \rightarrow \infty$ and so we have $d(x, z)=0$. This completes the proof.

\section{Application}

In this section, we suppose that $\left(E_{1},\|\cdot\|_{1}, \leq_{1}\right)$ is a partially ordered normed space with the following conditions:

(a) for all $x, y \in E_{1}, x \leq_{1} y \Longrightarrow r x \leq_{1} r y$ for all $r \in \mathbb{R}^{+}$;

(b) for all $x, y \in E_{1}$, there exists $z \in E_{1}$ such that $z$ is comparable to $x$ and $y$.

Also, we suppose that $\left(E_{2},\|\cdot\|_{2}, \leq_{2}\right)$ is a partially ordered Banach space with the condition (i) and satisfies the following:

(c) for all $x, y \in E_{1}$, there exists $z \in E_{1}$ such that $z$ is an upper bound of $\{x, y\}$;

(d) if $\left\{x_{n}\right\}$ is a nondecreasing sequence in $E_{2}$ and $x_{n} \rightarrow x$, then $x \geq x_{n}$ for all $n \in \mathbb{N}$.

As a simple example, we can show that $\mathbb{R}$ satisfies the conditions (a), (b), (c) and (d). Also, in this section, we consider $0 \times \infty=0$.

Now, we prove the main result of this section as follows.

Theorem 3.1 Suppose that $f: E_{1} \rightarrow E_{2}$ is a mapping satisfying

$$
2 f(x) \leq_{2} f(2 x)
$$

and

$$
\|f(x+y+z-w)-f(x)-f(y)-f(z)+f(w)\|_{2} \leq \phi(x, z)+\phi(y, w)
$$

for all $x, y, z, w \in E_{1}$, where $x$ is comparable to $z, y$ is comparable to $w$, where $\phi: E_{1} \times E_{1} \rightarrow$ $[0, \infty)$ is a function satisfying $\phi(0,0)=0$ and the following condition:

$$
\phi(x, y) \leq 2 L \phi\left(\frac{x}{2}, \frac{y}{2}\right)
$$

for all $x, y \in E_{1}$, where $x$ is comparable to $y$ and $L \in(0,1)$ is a constant. Then there exists a unique additive mapping $T: E_{1} \rightarrow E_{2}$ such that

$$
\|T(x)-f(x)\|_{2} \leq \frac{1}{1-L} \phi(x, x)
$$

for all $x \in E_{1}$.

Proof It is clear that $f(0)=0$. Putting $z:=x$ and $y=w:=0$ in (3.2), we get

$$
\|f(2 x)-2 f(x)\|_{2} \leq \phi(x, x)
$$


for all $x \in E_{1}$. Hence we have

$$
\left\|\frac{f(2 x)}{2}-f(x)\right\|_{2} \leq \frac{1}{2} \phi(x, x) \leq \phi(x, x)
$$

for all $x \in E_{1}$. Consider $X:=\left\{g: g: E_{1} \rightarrow E_{2}\right\}$ and introduce the generalized metric $d$ on $X$ by

$$
d(h, g):=\inf \left\{C \in \mathbb{R}^{+}:\|h(x)-g(x)\|_{2} \leq C \phi(x, x), \forall x \in E_{1}\right\}
$$

for all $h, g \in X$. It is easy to show that $(X, d)$ is a complete generalized metric space.

Now, we put the partial order $\leq$ on $X$ as follows: for all $h, g \in X$,

$$
h \leq g \quad \Longleftrightarrow \quad h(x) \leq_{2} g(x)
$$

for all $x \in E_{1}$. Now, we define a mapping $J: X \rightarrow X$ by

$$
J(h)(x):=\frac{1}{2} h(2 x)
$$

for all $x \in E_{1}$. For any $g, h \in X$ with $g \leq h$, it follows that, for all $x \in E_{1}$,

$$
\begin{aligned}
d(g, h)<C & \Longrightarrow\|g(x)-h(x)\|_{2} \leq C \phi(x, x) \\
& \Longrightarrow\left\|\frac{g(2 x)}{2}-\frac{h(2 x)}{2}\right\|_{2} \leq C \frac{\phi(2 x, 2 x)}{2} \\
& \Longrightarrow\|J(g)(x)-J(h)(x)\|_{2} \leq L C \phi(x, x) .
\end{aligned}
$$

It follows that

$$
d(J(g), J(h)) \leq L d(g, h) .
$$

It is easy to show that $J$ is a nondecreasing mapping.

Now, we show that $J$ is a continuous function. To this end, let $\left\{h_{n}\right\}$ be a sequence in $(X, d)$ which converges to $h \in X$ and let $\epsilon>0$ be given. Then there exist $N \in \mathbb{N}$ and $C \in \mathbb{R}^{+}$with $C \leq \epsilon$ such that

$$
\left\|h_{n}(x)-h(x)\right\|_{2} \leq C \phi(x, x)
$$

for all $x \in E_{1}$ and $n \geq N$ and so

$$
\left\|h_{n}(2 x)-h(2 x)\right\|_{2} \leq C \phi(2 x, 2 x)
$$

for all $x \in E_{1}$ and $n \geq N$. By inequality (3.3) and the definition of $J$, we get

$$
\left\|J\left(h_{n}\right)(x)-J(h)(x)\right\|_{2} \leq L C \phi(x, x)
$$


for all $x \in E_{1}$ and $n \geq N$. Hence

$$
d\left(J\left(h_{n}\right), J(h)\right) \leq L C<\epsilon
$$

for all $n \geq N$. It follows that $J$ is continuous. On the other hand, by (3.1), we have $f \leq J(f)$ and, by applying inequality (3.5), we see that $d(J(f), f) \leq 1$. Applying Theorem 2.1 , it follows that $J$ has a fixed point $T \in X$ such that $\lim _{n \rightarrow \infty} d\left(J^{n}(f), T\right)=0$. It follows that

$$
T(x)=\lim _{n \rightarrow \infty} \frac{f\left(2^{n} x\right)}{2^{n}}
$$

for all $x \in E_{1}$. For any $x \in E_{1}$, it follows from (3.1) that the sequence $\left\{\frac{f\left(2^{n} x\right)}{2^{n}}\right\}$ is a nondecreasing sequence in $E_{2}$ and so, by (3.6), we have $\frac{f\left(2^{n} x\right)}{2^{n}} \leq T(x)$ for all $n \geq 0$. In particular, $f(x) \leq T(x)$. This shows that $f \leq T$. Now, we can see that

$$
d(J(f), J(T)) \leq L d(f, T)
$$

and hence

$$
d(f, T) \leq \frac{1}{1-L} .
$$

This implies inequality (3.4).

On the other hand, by using inequality (3.3), we have

$$
\phi\left(2^{n} x, 2^{n} y\right) \leq L^{n} \phi(x, y)
$$

for all $x, y \in E_{1}$ and $n \in \mathbb{N}$, where $x$ is comparable to $y$. Let $x, y \in E_{1}$ be arbitrary elements. Then there exists $z \in E_{1}$ such that $z$ is comparable to $x$ and $y$. This implies that $2^{n} z$ is comparable to $2^{n} x$ and $2^{n} y$ for all $n \in \mathbb{N}$. It follows from (3.2) that

$$
\begin{aligned}
& \left\|f\left(2^{n}(x+y)\right)-f\left(2^{n} x\right)-f\left(2^{n} y\right)\right\|_{2} \\
& \quad=\left\|f\left(2^{n} x+2^{n} y+2^{n} z-2^{n} z\right)-f\left(2^{n} x\right)-f\left(2^{n} y\right)-f\left(2^{n} z\right)+f\left(2^{n} z\right)\right\|_{2} \\
& \quad \leq \phi\left(2^{n} x, 2^{n} z\right)+\phi\left(2^{n} y, 2^{n} z\right)
\end{aligned}
$$

for all $n \in \mathbb{N}$. By using (3.6) and (3.7), it follows that $T$ is a Cauchy mapping.

To prove the uniqueness property of $T$, suppose that $T_{1}$ is another additive function satisfying (3.4). It is clear that $J\left(T_{1}\right)=T_{1}$. Then, for any $x \in E_{1}$, there exists $g(x) \in E_{2}$ such that $g(x)$ is an upper bound of $\left\{T(x), T_{1}(x)\right\}$. This shows that $g: E_{1} \rightarrow E_{2}$ is a mapping which is comparable to $T$ and $T_{1}$. Hence we have

$$
\begin{aligned}
d\left(T, T_{1}\right) & \leq d\left(T, J^{n}(g)\right)+d\left(J^{n}(g), T_{1}\right) \\
& =d\left(J^{n}(T), J^{n}(g)\right)+d\left(J^{n}(g), J^{n}\left(T_{1}\right)\right) \\
& \leq L^{n} d(T, g)+L^{n} d\left(g, T_{1}\right)
\end{aligned}
$$

for all $n \in \mathbb{N}$. Since $L<1, T=T_{1}$. This completes the proof. 
Corollary 3.2 Let $\epsilon \in[0, \infty)$ and $f: E_{1} \rightarrow E_{2}$ be a function such that $f(0)=0$ and

$$
2 f(x) \leq_{2} f(2 x)
$$

and

$$
\|f(x+y+z-w)-f(x)-f(y)-f(z)+f(w)\|_{2} \leq \epsilon
$$

for all $x, y, z, w \in E_{1}$, where $x$ is comparable to $z$ and $y$ is comparable to $w$. Then there exists a unique additive mapping $T: E_{1} \rightarrow E_{2}$ such that

$$
\|T(x)-f(x)\|_{2} \leq \epsilon
$$

for all $x \in E_{1}$.

Proof Set $\phi(x, y)=\frac{\epsilon}{2}$ for all $x, y \in E_{1}$ with $x, y \neq 0, \phi(0,0)=0$, and let $L=\frac{1}{2}$ in Theorem 3.1. Then we get the desired result.

Corollary 3.3 Let $p \in(0,1)$ and $\epsilon \in[0, \infty)$. Suppose that $f: E_{1} \rightarrow E_{2}$ is a mapping such that

$$
2 f(x) \leq_{2} f(2 x)
$$

and

$$
\|f(x+y+z-w)-f(x)-f(y)-f(z)+f(w)\|_{2} \leq \epsilon\left(\|x\|^{p}+\|y\|^{p}+\|z\|^{p}+\|w\|^{p}\right)
$$

for all $x, y, z, w \in E_{1}$, where $x$ is comparable to $z$ and $y$ is comparable to $w$. Then there exists a unique additive mapping $T: E_{1} \rightarrow E_{2}$ such that

$$
\|T(x)-f(x)\|_{2} \leq \frac{2^{2-p}}{2^{1-p}-1} \epsilon\|x\|^{p}
$$

for all $x \in E_{1}$.

Proof Set $\phi(x, y)=\epsilon\left(\|x\|^{p}+\|y\|^{p}\right)$ for all $x, y \in E_{1}$, and let $L=2^{p-1}$ in Theorem 3.1. Then we get the desired result.

\section{Competing interests}

The authors declare that they have no competing interests.

\section{Authors' contributions}

All authors read and approved the final manuscript.

\section{Author details}

'Department of Mathematics, Semnan University, P.O. Box 35195-363, Semnan, Iran. ${ }^{2}$ Department of Mathematics Education and the RINS, Gyeongsang National University, Chinju, 660-701, Korea. ${ }^{3}$ Department of Mathematics and Research Institute for Natural Sciences, Hanyang University, Seoul, 133-791, Korea. 


\section{Acknowledgements}

YJ Cho and C Park were supported by the Basic Science Research Program through the National Research Foundation of Korea funded by the Ministry of Education, Science and Technology (NRF-2012-0008170) and (NRF-2012R1A1A2004299), respectively.

Received: 20 May 2013 Accepted: 29 October 2013 Published: 20 Jan 2014

\section{References}

1. Ulam, SM: Problems in Modern Mathematics, Chapter VI, Science Ed. Wiley, New York (1940)

2. Hyers, DH: On the stability of the linear functional equation. Proc. Natl. Acad. Sci. USA 27, $222-224$ (1941)

3. Diaz, JB, Margolis, B: A fixed point theorem of the alternative for contractions on a generalized complete metric space. Bull. Am. Math. Soc. 74, 305-309 (1968)

4. Nieto, JJ, Rodriguez-Lopez, R: Contractive mapping theorems in partially ordered sets and applications to ordinary differential equations. Order 22, 223-239 (2005)

5. Cădariu, L, Radu, V: Fixed points and the stability of Jensen's functional equation. J. Inequal. Pure Appl. Math. 4(1), Article ID 4 (2003)

6. Cădariu, L, Gavruta, L, Gavruta, P: Weighted space method for the stability of some nonlinear equations. Appl. Anal. Discrete Math. 6, 126-139 (2012)

7. Cădariu, L, Gavruta, L, Gavruta, P: Fixed points and generalized Hyers-Ulam stability. Abstr. Appl. Anal. 2012, Article ID $712743(2012)$

8. Eshaghi Gordji, M, Najati, A: Approximately J*-homomorphisms: a fixed point approach. J. Geom. Phys. 60, 809-814 (2010)

9. Jung, S, Rassias, JM: A fixed point approach to the stability of a functional equation of the spiral of Theodorus. Fixed Point Theory Appl. 2008, Article ID 945010 (2008)

10. Brzdek, J, Chudziak, J, Pales, Z: A fixed point approach to stability of functional equations. Nonlinear Anal. 74, 6728-6732 (2011)

11. Brzdek, J, Cieplinski, K: A fixed point approach to the stability of functional equations in non-Archimedean metric spaces. Nonlinear Anal. 74, 6861-6867 (2011)

12. Brzdek, J, Cieplinski, K: A fixed point theorem and the Hyers-Ulam stability in non-Archimedean spaces. J. Math. Anal. Appl. 400, 68-75 (2013)

13. Nieto, JJ, Pouso, RL, Rodriguez-Lopez, R: Fixed point theorems in ordered abstract spaces. Proc. Am. Math. Soc. 135 2505-2517 (2007)

14. Dinevari, T, Frigon, M: Fixed point results for multivalued contractions on a metric space with a graph. Proc. Am. Math. Soc. 135, 2505-2517 (2013)

15. Nieto, JJ, Rodriquez-Lopez, R: Existence and uniqueness of fixed point in partially ordered sets and applications to ordinary differential equations. J. Math. Anal. Appl. 405, 507-517 (2007)

16. Paesano, D, Vetro, P: Suzuki's type characterizations of completeness for partial metric spaces and fixed points for partially ordered metric spaces. Topol. Appl. 159, 911-920 (2012)

10.1186/1687-1812-2014-15

Cite this article as: Eshaghi Gordji et al.: A new type fixed point theorem for a contraction on partially ordered generalized complete metric spaces with applications. Fixed Point Theory and Applications 2014, 2014:15

\section{Submit your manuscript to a SpringerOpen ${ }^{\circledR}$ journal and benefit from:}

- Convenient online submission

- Rigorous peer review

- Immediate publication on acceptance

- Open access: articles freely available online

- High visibility within the field

- Retaining the copyright to your article 